Ann. Biol. anim. Bioch. Biophys., 1979, 19 (1B), 207-215.

\title{
Etude expérimentale du développement fœtal dans la déficience en acide folique induite par antivitamines (Acide X-méthyl-folique, Méthotrexate)
}

\author{
par Geneviève POTIER de COURCY, Thérèse TERROINE \\ avec la collaboration de Sylvie DESMETTRE-MIGUET, J. Ph. CHRISTIDES \\ Cenfre de Recherches sur la Nutrition, C.N.R.S. \\ 9, rue Jules-Hetzel, 92190 Meudon-Bellevue.
}

Summary. Fefal development under experimental folic acid deficiency induced by methotrexate or X-methyl-folic acid.

Tritium-labelled folic acid $(10 \mu \mathrm{Ci} / 100 \mathrm{~g})$ was given i. p. to normal pregnant rats to study vitamin transfer from mother to fetus. Between days 13 and 21 of pregnancy, uptake of radioactivity $/ g$ in the latter varied from 0.86 to $1.09 \mathrm{p} .100$ of the dose injected to the mother as the amount of folates increased 3.5-fold during that time. $X$-methyl-folic acid was given by oral route ( 0.5 p. 100 of the diet on days 11 and 12 of pregnancy). Many fetal malformations were obtained, associated with a marked undergrowth. Significantly lower amounts of folates were found in the group of smaller malformed fetuses. Methotrexate (Mtx) was injected i. p. to pregnant rats 3 days between days 7 and 16 of pregnancy in doses varying from 1.5 to $60 \mu \mathrm{g} / 100 \mathrm{~g} /$ day. Mortality was maximal with doses of Mtx between 10 and $14 \mu \mathrm{g}$ injected between days 7 and 11 . Hypotrophy was maximal with 50 and $60 \mu \mathrm{g}$ of $\mathrm{Mtx}$ injected between days 14 to 16 . In each case, the incidence of morphological anomalies was very low. It is suggested from these data that folates might be stored in the fetus during the second half of pregnancy when maternal nutritional state is optimal. Folic acid antagonists prevented this storage, even when growth was apparently normal.

\section{Introduction.}

Les antimétabolites de l'acide folique sont tératogènes pour le fœtus humain, que ce soit l'aminoptérine ou acide 4-amino-ptéroyl-glutamique (Thiersch, 1952), ou l'améthoptérine ou méthotrexate (acide 4-amino-10-méthyl-ptéroyl-glutamique) (Powell et Ekert, 1971). Ils le sont également pour l'cirimal mais les études expérimentales dans ce domaine portent surtout sur l'acide $X$-méthyl-folique (Nelson ef al., 1955) et l'acide 9-méthyl-folique (Johnson ef al., 1963). Ils sont administrés par voie orale. Nous avons nous-mêmes obtenu avec l'acide $X$-méthyl-folique un grand nombre d'anomalies chez le Rat, dont la plupart localisées à la face et aux extrémités (Potier de Courcy, 1968). 
Dans le but d'obtenir des anomalies de même nature par une antivitamine purifiée et donc injectable, nous avons choisi le méthotrexate en le donnant à la ratte gestante à des stades plus tardifs que ceux utilisés par d'autres auteurs (Baranov, 1966 ; Wilson, 1970) pour nous situer dans des conditions comparables à celles employées avec l'acide $X$-méthyl folique. L'étude systématique des effets de l'injection de l'antivitamine a été faite en fonction de la dose et de la période de la gestation. Poursuivant simultanément notre étude sur les effets de l'acide $X$-méthyl folique, nous avons recherché l'existence d'une relation entre le poids du fœtus à la veille de la naissance, la quantité d'acide folique ef la présence des malformations.

Parallèlement a été étudiée chez le fœetus témoin la dynamique du dépôt de la vitamine en fin de gestation par le dosage des folates dans la portée et la rétention globale du traceur radioactif par le foetus au cours de la deuxième moitié de la gestation. On sait que dans l'espèce humaine le nouveau-né dispose d'une forte réserve en folates, atfestée par sa forte teneur érythrocytaire (Navarro, 1973). Une analogie possible avec l'Homme, consfatée dans plusieurs autres cas — tératogenèse par les antivitamines, importance déterminante des enzymes du cycle folique chez le foetus (Gaull ef al., 1973) - nous poussait à rechercher si au niveau du stockage également le Rat pouvait constituer un bon modèle expérimental.

\section{Matériel ef méthodes.}

Animaux. - Les rattes sont de souche Wistar AG ; elles proviennent de l'élevage du CNRS et ont de 220 à $230 \mathrm{~g}$ au moment de l'accouplement. Cycles et accouplement sont déterminés par frottis vaginal.

Régimes. - lls sont à base de caséine dévitaminée NBC (25 g/100 g), d’huile d'arachide $(10 \mathrm{~g} / 100 \mathrm{~g})$; de saccharose $(58 \mathrm{~g} / 100 \mathrm{~g})$, de cellulose $(2 \mathrm{~g} / 100 \mathrm{~g})$ et de mélange salin HMW $(4 \mathrm{~g} / 100 \mathrm{~g})$ et vitaminique NBC $(1 \mathrm{~g} / 100 \mathrm{~g})$. Ils renferment en outre 1 p. 100 de succisulfathiazol Théraplix, pour éviter la synthèse intestinale d'acidefolique par la flore, et lorsque nécessaire, 0,5 p. 100 d'acide $X$-méthyl-folique les $11 \mathrm{e}$ et $12^{\circ}$ jours de la gestation. Le régime témoin contient $5 \mathrm{mg}$ par $\mathrm{kg}$ d'acide folique.

Traitement. - Le méthotrexate est donné par injection intra-péritonéale par période de 3 jours, à une dose pouvant aller de $1,5 \mu \mathrm{g} / 100 \mathrm{~g} / \mathrm{jour}$ à 60 , entre le $7 \mathrm{e}$ ef le $16 \mathrm{e}$ jour de la gestation.

Les dosages de folates sont faits par microbiologie, à l'aide de L. Casei selon la méthode classique de Herbert (1966) après déconjugaison par autolyse.

Pour le traçage radioactif, on utilise l'acide $(3 \mathrm{H}) 3^{\prime}-5^{\prime}$ folique (activité spécifique : $25 \mathrm{Ci} / \mathrm{mM}$ ), par injection intra-péritonéale, à la dose de $10 \mu \mathrm{Ci} / 100 \mathrm{~g}$ d'animal : les prélèvements et comptages ont lieu $24 \mathrm{~h}$ après, les essais préliminaires nous ayant montré que dans le fotus l'équilibre isotopique était pleinement atteint à ce moment-là.

Les sacrifices ont lieu par décapitation, du 13 au $21^{\mathrm{e}}$ jour de la gestation selon le cas, les implantations ef résorptions dénombrées, les embryons ou fœetus vivants examinés et pesés individuellement frès rapidement. 


\section{Résultats et discussion.}
A. Etude du dépôt des folates dans le foetus témoin.

Au cours de la deuxième moitié de la gestation (13e-21e jour), le dosage microbiologique des folates par $L$. Casei après déconjugaison dans le fœetus montre que la concentration par gramme passe de $752 \mu \mathrm{g}$ à 2401 pendant cette période (tabl. 1). Il y a donc manifestement accumulation de cet élément par unité de poids avant la naissance, accumulation qui se traduit au niveau du fotus entier par un dépôt considérable, puisque la quantité de folates totaux du corps foetal passe de 17,2 $\pm 2,3 \mu \mathrm{g}$ d'acide folique à $7750 \pm 593$ (tabl. 1).

\section{TABLEAU 1}

Folates totaux (dosés par L. Casei après autolyse) trouvés dans le fœilus au cours de la dernière période de la gestation (13-21e jour)

\begin{tabular}{|c|c|c|c|c|}
\hline \multirow{2}{*}{ Stade de la gestation } & \multirow{2}{*}{$\begin{array}{c}\text { Nombre } \\
\text { de données } \\
\text { (nombre fotal } \\
\text { de fœetus dosés) }\end{array}$} & \multirow{2}{*}{$\begin{array}{l}\text { Poids moyen } \\
\text { du fœetus } \\
\text { (mg) }\end{array}$} & \multicolumn{2}{|c|}{ Folates } \\
\hline & & & $\mu g / g$ & $\mu g / f œ f u s$ \\
\hline $13 \mathrm{e}$ jour...$\ldots \ldots \ldots$ & $\begin{array}{c}8 \\
(46)\end{array}$ & $24 \pm 1$ & $752 \pm 134$ & $17 \pm 2$ \\
\hline $15^{e}$ jour & $\begin{array}{l}10 \\
(46)\end{array}$ & $155 \pm 6$ & $775 \pm 168$ & $122 \pm 29$ \\
\hline 17 jour ............. & $\begin{array}{l}11 \\
(48)\end{array}$ & $412 \pm 6$ & $1142 \pm 86$ & $469 \pm 36$ \\
\hline $19 \mathrm{e}$ jour...$\ldots \ldots \ldots$ & $\begin{array}{l}15 \\
(51)\end{array}$ & $1319 \pm 23$ & $1293 \pm 234$ & $1861 \pm 322$ \\
\hline $21^{e}$ jour...$\ldots \ldots \ldots$ & $\begin{array}{l}18 \\
(18)\end{array}$ & $3289 \pm 93$ & $2401 \pm 186$ & $7750 \pm 593$ \\
\hline
\end{tabular}

L'injection du traceur radioactif à la mère (acide $(3 \mathrm{H})$-folique, $10 \mu \mathrm{Ci} / 100 \mathrm{~g}$, i. p.) permet de voir que la quantité de radioactivité retenue par $24 \mathrm{~h}$ dans le fotus par rapport à la dose injectée est seulement en faible augmentation pendant la période considérée (13e-21e jour), lorsque cette valeur est rapportée à l'unité de poids fœetal, puisqu'elle représente 0,86 p. 100 au 13 jour et 1,09 p. 100 à la veille de la naissance (łabl. 2). Evaluée par fœetus entier, la radioactivité totale retenue par $24 \mathrm{~h}$ passe de $0,018 \pm 0,002$ à $3,53 \pm 0,23$ au cours de ces huit jours, en p. 100 de la dose injectée. De ces résultats, on peut déduire que s'il n'y a pas augmentation notable du dépôt de folates par unité de poids dans le fœitus, la quantité totale de vitamine transférée de la mère aux fœius représente cependant une valeur très importante : pour l'ensemble de la portée, la captation totale du traceur, toujours pour $24 \mathrm{~h}$, par rapport à la dose injectée, s'élève à 40 p. 100 au $21 \mathrm{e}$ jour de la gestation alors qu'elle n'est que de 0,216 au 13 jour. Une exploration au niveau de l'excrétion urinaire du traceur par la mère ef sa rétention par le foie nous a montré (résultats non publiés) que la femelle gravide compense en partie cette forte « fuite » de folates vers la portée en fin de gestation par 
une diminution de l'excrétion urinaire des folates et de leur dépôł dans son foie : la radioactivité retenue par $24 \mathrm{~h}$ passe en effet de 25 à 15 p. 100 de la dose reçue entre le $13^{e}$ et le $21^{\text {e }}$ jour dans le cas du foie et la quantité excrétée de 34 à 21 p. 100.

TABLEAU 2

Radioactivité totale retenue par le fœitus en $24 \mathrm{~h}$ après injection i. p. d'acide folique tritié à la mère $(10 \mu \mathrm{Ci} / 100 \mathrm{~g})$. Période du 13 au $21^{\mathrm{e}}$ jour

\begin{tabular}{|c|c|c|c|c|c|}
\hline \multirow{2}{*}{$\begin{array}{c}\text { Stade } \\
\text { de la gestation } \\
\text { (nombre } \\
\text { de données) }\end{array}$} & \multirow{2}{*}{$\begin{array}{r}\text { Nombre de } \\
\text { foetus/portée }\end{array}$} & \multirow{2}{*}{$\begin{array}{c}\text { Poids moyen } \\
\text { du fœtus } \\
\text { (mg) }\end{array}$} & \multicolumn{3}{|c|}{ Radioactivité (en p. 100 de la dose injectée) } \\
\hline & & & $/ 9$ & foetus & /portée \\
\hline $\begin{array}{l}13^{\mathrm{e}} \text { jour } \ldots \ldots \\
\quad(7)\end{array}$ & $14,3 \pm 0,8$ & $21 \pm 2$ & $0,86 \pm 0,10$ & $0,018 \pm 0,002$ & $0,216 \pm 0,0016$ \\
\hline $\begin{array}{c}15 \mathrm{e} \text { jour } \ldots \ldots \ldots \\
(6)\end{array}$ & $11,5 \pm 1,3$ & $136 \pm 8$ & $0,66 \pm 0,06$ & $0,089 \pm 0,080$ & $0,910 \pm 0,210$ \\
\hline $17 \mathrm{e}$ jour ......... & $12,0 \pm 1,8$ & $422 \pm 17$ & $0,78 \pm 0,03$ & $0,326 \pm 0,008$ & $3,65 \pm 0,77$ \\
\hline $\begin{array}{c}19 \mathrm{e} \text { jour ........ } \\
(7)\end{array}$ & $14,5 \pm 0,3$ & $1250 \pm 20$ & $0,94 \pm 0,12$ & $1,18 \pm 0,13$ & $\pm 1,6$ \\
\hline$\underset{(6)}{21 \mathrm{e} \text { jour } . . . . . . . ~}$ & $12,7 \pm 0,8$ & $3280 \pm 70$ & $1,09 \pm 0,08$ & $3,53 \pm 0,23$ & $40,3 \pm 3,3$ \\
\hline
\end{tabular}

B. - Effets des antivitamines : acide $X$-méthyl-folique ef méthotrexate.

La carence maternelle par l'acide X-méthyl-folique est obtenue par administration alimentaire de l'antivitamine les $11^{\mathrm{e}}$ et $12^{\mathrm{e}}$ jours de la gestation à la dose de 0,5 p. 100 du régime. Malgré un régime identique et un appétit normal chez toutes les mères de ce groupe, la réponse des fœetus va d'un développement apparemment normal à l'apparition des anomalies les plus profondes de la face et des membres (bec-de-lièvre, fente de la face, absence de paupières, pied-bot, absence ou torsion de la queue, Potier de Courcy, 1968), selon les portées ef à l'intérieur des mêmes portées. La déficience maternelle se caractérise en outre par une hypotrophie marquée chez les fœtus ef par un grand nombre de résorptions. En divisant les fatus en 3 catégories : témoins, carencés non malformés et carencés malformés, nous avons dosé les folates déposés dans les fotus de chaque groupe au $21^{\mathrm{e}}$ jour afin de rechercher une relation entre quantité de folates déposés en fin de gestation et présence de malformations d'une part, quantité de folates et niveau de croissance, d'autre part.

En portant la quantité totale de folates du fœtus en ordonnée ef le poids des fœius en abscisse, on obtient une courbe ininterrompue entre les témoins et les carencés et qui présente la caractéristique d'être asymptotique aux deux extrémités (fig. 1). Cela pourrait signifier, en ce qui concerne le groupe des témoins, qu'au-delà d'un poids moyen optimum atteint dans les portées de mères non limitées en acide folique, les fœtus disposent d'une quantifé de folates supérieure aux besoins de la croissance et se montrent capables de les accumuler. Cette observation va dans le même sens que les résultats concernant la dynamique de dépôt des folates en fin de gestation qui 
faisaient état d'une très forte accumulation de la vitamine dans les jours précédant la naissance.

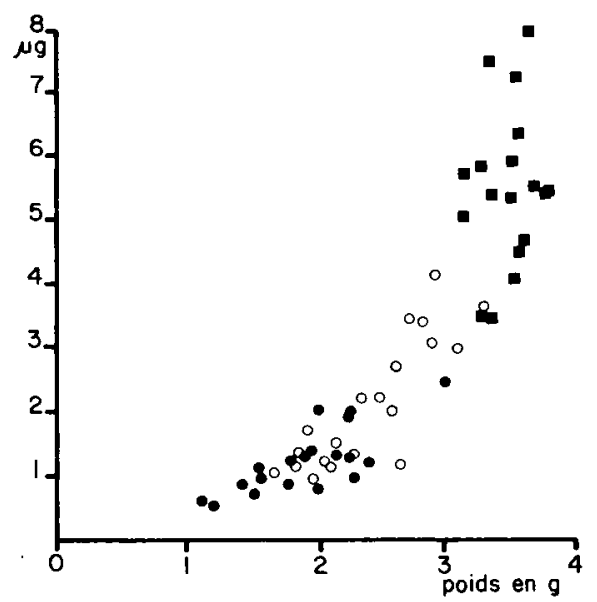

FIG. 1. - Folates totoux du fœetus dosés par L. cosei après autolyse. Teneur par fœetus.

\section{- témoins}

- carencés ( ${ }^{1}$ ) non malformés

- carencés malformés,

(1) La carence est obtenue par l'acide $X$-méthyl-folique (0,5 p. 100 du régime, les 11 et $12^{\mathrm{e}}$ jours de la gestation).

A l'inverse, en deçà d'une certaine quantité de folates que nous évaluons graphiquement à $0,5 \mu \mathrm{g} /$ fotus au $21^{\mathrm{e}}$ jour, il y a mort de l'embryon aux stades antérieurs ; en dessous d'une autre valeur située, toujours au $21^{\mathrm{e}}$ jour, autour de $1,28 \mu \mathrm{g}$, il y a présence presque systématique d'anomalies morphologiques (tabl. 3) et une hypotrophie drastique. Enfin, lorsque les folates augmentent en quantité, les anomalies apparentes disparaissent mais la croissance est une fonction (non linéaire) de leur valeur dans le fœtus (fig. 1) : autrement dit, la quantité de folates présente dans le fœus semble bien être le facteur limitant de sa croissance.

TABLEAU 3

Folates totaux (dosés par L. Casei après autolyse) dans les foetus de 20 jours témoins et carencés en acide folique *

\begin{tabular}{|c|c|c|}
\hline Lots (nombre de données) & $\begin{array}{l}\text { Poids moyen } \\
\text { du fœetus } \\
\text { (g) }\end{array}$ & $\begin{array}{l}\text { Folates } \\
\text { ( } \mu \mathrm{g} / \text { fotus) }\end{array}$ \\
\hline $\begin{array}{l}\text { Témoins }(20) \ldots \ldots \ldots \ldots \ldots \ldots \ldots \ldots \\
\text { Carencés }\end{array}$ & $3,34^{a} \pm 0,066^{* *}$ & $5,88^{a} \pm 0,40$ \\
\hline $\begin{array}{l}\text { non malformés }(21) \ldots \ldots \ldots \ldots \ldots \\
\text { malformés }(21) \ldots \ldots \ldots \ldots \ldots\end{array}$ & $\begin{array}{l}2,44 \pm 0,095 \\
1,93^{\mathrm{a}} \pm 0,097\end{array}$ & $\begin{array}{l}2,11 \mathrm{ab} \pm 0,22 \\
1,28 \mathrm{ab} \pm 0,10\end{array}$ \\
\hline
\end{tabular}

* La mère a reçu dans le régime 0,5 p. 100 d'acide $X$-méthylfolique les $11 \mathrm{e}$ et $12^{\mathrm{e}}$ jours de gestation. ** Les valeurs suivies de la même lettre sont significativement différentes. 
Nous avons cherché à obtenir des anomalies fœetales avec le méthotrexate dans des conditions comparables à celles utilisées avec l'acide-X-méthyl folique. Les essais ont donc surtout été menés autour du $10 \mathrm{e}$ jour de la gestation.

L'analogie de réponse entre un certain nombre de lots traités au méthotrexate nous ont permis de les regrouper en trois grandes catégories, selon les périodes d'injection : du $7 \mathrm{e}$ au $11^{\mathrm{e}}$ jour, du $10^{\mathrm{e}}$ au $13^{\mathrm{e}}$ jour et $\mathrm{du} 12^{\mathrm{e}}$ au $16^{\mathrm{e}}$ jour.

Le faux de résorptions montre que plus l'embryon est jeune, plus sa sensibilité à l'antivitamine est aiguë : on a 100 p. 100 de résorptions avec des doses de 14 à $16 \mu \mathrm{g}$ injectées entre le $7 \mathrm{e}$ et le $11 \mathrm{e}$ jour alors qu'il n'y en a plus que $66 \mathrm{p} .100$ avec ces doses du $10^{\mathrm{e}}$ au $13^{\mathrm{e}}$ jour ef 11 p. $100 \mathrm{du} 12^{\mathrm{e}}$ au $16^{\mathrm{e}}$ jour (tabl. 4).

Le poids fotal est une autre caractéristique des effets des antimétabolites étudiés : nous avons obtenu une baisse de croissance fœtale très nette ( -15 p. 100) chez les survivants du premier groupe, 20 fœtus sur 33 ayant moins de $3 \mathrm{~g}$ au $21 \mathrm{e}$ jour. L'hypotrophie apparaît beaucoup moins nette dans le groupe intermédiaire, quelle que soit la fréquence des résorptions, mais redevient forte en période d'injection tardive avec une forte dose d'antivitamine (-31 p. 100) (tabl. 4).

En somme, la zone de sensibilité maximale aux résorptions se situe au début de la

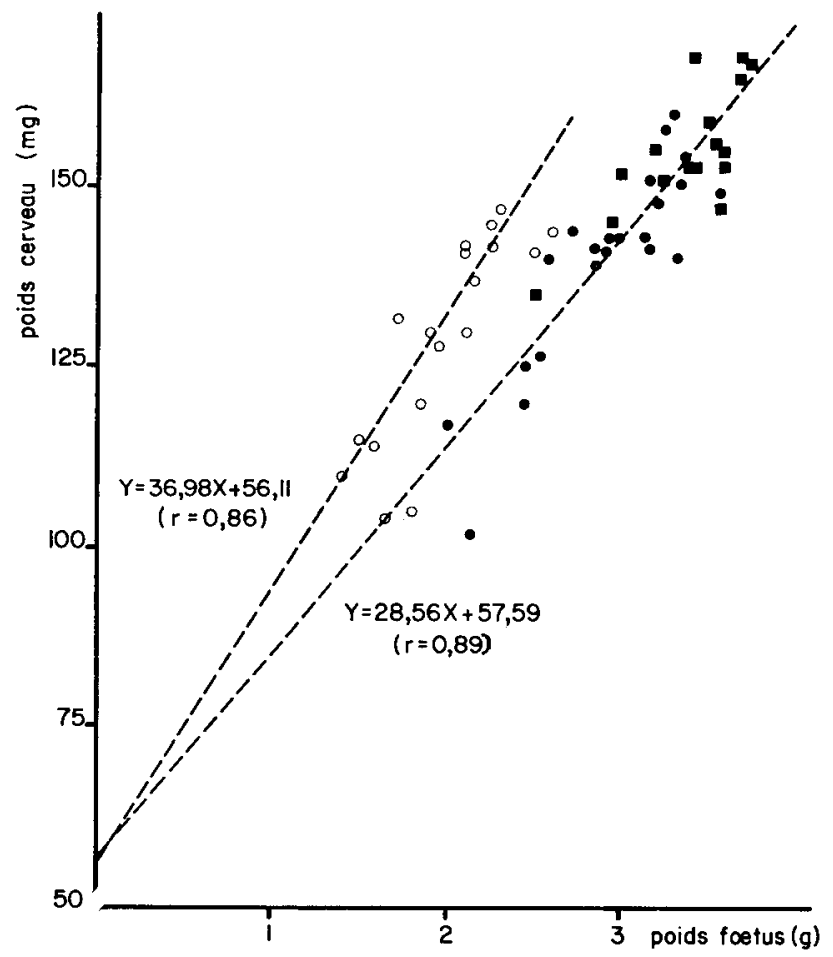

FIG. 2. - Effet de l'injection du méthotrexate à la mère sur la relation poids du cerveou fœial-poids du corps.

\footnotetext{
- témoins

- carencés 10 à $40 \mu \mathrm{g}$ de méthotrexate en milieu de gestation (entre le 7 et le $15^{\circ}$ jour)

- carencés 50 à $60 \mu \mathrm{g}$ de méthotrexate en fin de gestation $\left(14,15\right.$ ef $16^{\mathrm{e}}$ jours).
} 


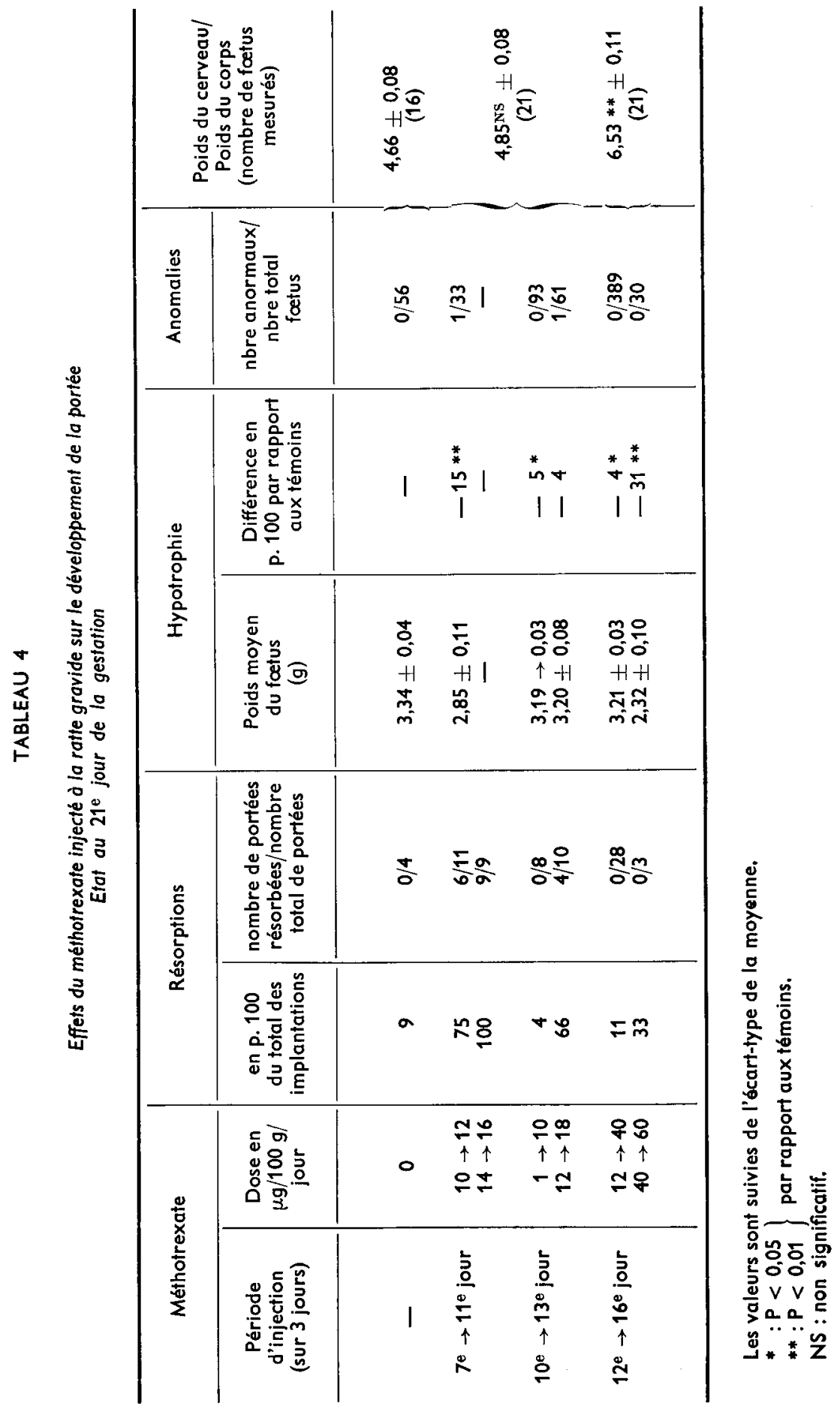


vie utérine alors qu'elle se situe plutôt dans la deuxième moitié de la gestation en ce qui concerne la croissance.

Enfin, pour ce qui est des anomalies, elles sonf pratiquement inexistantes (tabl. 4). Une certaine inhibition dans le rythme de croissance des embryons reste donc la principale caractéristique des effets du méthotrexate administré après le 7 e jour de la gestation.

En essayant d'établir une corrélation entre le poids du cerveau et le poids du corps pour évaluer les différences possibles dues au traitement, nous avons observé que les points concernant le lot témoin étaient définis par une même fonction linéaire que ceux des lots carencés en acide folique, excepté pour les individus dont la mère avait reçu 50 ef $60 \mu \mathrm{g}$ d'antivitamine du $14^{\mathrm{e}}$ au $16^{\mathrm{e}}$ jour qui se sifuent sur une autre droite dont l'origine est identique mais la pente significativement différente (fig. 2). Nous en déduisons qu'à partir de ce stade, le méthotrexate inhibe relativement moins la croissance du cerveau que celle du reste de l'organisme.

\section{Conclusions.}

L'étude du transfert de l'acide folique vers le fœtus avec une alimentation maternelle excédentaire en cette viłamine $(5 \mathrm{mg} / \mathrm{kg}$ de régime) montre que le rythme de captation du traceur radioactif (acide $3 \mathrm{H}-3^{\prime}-5^{\prime}$ folique) par le fœtus est relativement faible par unité de poids au cours du dernier tiers de la gestation chez le Rat. Dans un essai comparable effectué chez la Femme enceinte, Landon et al. (1975) s'étonnent que moins de 1 p. 100 seulement de la radioactivité administrée à la mère se retrouve dans le produit de la conception. Dans notre cas cependant, la demande totale d'acide folique de l'ensemble de la protée représente une très forte quantité ef donc un besoin considérable en cette vitamine pour la mère en fin de gestation. Le fait que les folates s'accumulent davantage avant la naissance dans le fotus que le rythme de rétention par $\mathrm{g}$ du traceur ne pourrait le laisser supposer montre bien que le fotus met une grande partie des folates qui lui parvient en réserve et cela quels que soient leur degré d'oxydation ou la longueur de leur chaîne en résidus glutamates (Potier de Courcy et Bertaux, 1972).

Lors d'ingestion d'une antivitamine par la mère, dans notre cas l'acide X-méthylfolique, on observe une chute considérable des folates du fœtus pouvant aller jusqu'au $1 / 10^{e}$ de la valeur moyenne trouvée chez les témoins dans le cas de fœtus hypotrophiques malformés. Il est probable que le déficit se situe au niveau de l'apport, les folates disponibles chez la mère étant mobilisés pour les besoins de la compétition enzymatique imposée par l'antivitamine. En tout état de cause, la quantité de folates présente dans le fœtus apparaît d'après nos résultats comme limitante pour la croissance du foetus même non malformé.

Le méthotrexate, éłudié ici en particulier pour ses potentialités tératogènes au cours des deux derniers tiers de la gestation, s'est révélé essentiellement être un agent embryoléthal ou hypotrophique selon le stade d'injection et la dose considérés. Cela est parfaitement en accord avec les derniers résultats de Jordan ef al. (1977) et ceux de Baranov (1966) et Wilson (1970) qui n'obtiennent en fait d'anomalies que dans les tous premiers stades de la gestation, avant la nidation. Dans notre cas, l'hypotrophie est 
d'autant plus forte et la mortalité embryonnaire faible que l'injection d'antivitamine est administrée à un stade plus tardif de la gestation. En fin de gestation et à forte dose, on observe une moindre sensibilité du cerveau au méthotrexate par rapport à la croissance globale du fœtus. Cette donnée est à rapprocher de certains de nos résultats antérieurs qui montraient qu'avec l'acide $X$-méthyl-folique, la croissance fœetale globale était abaissée de 41 p. 100 alors que celle du cerveau l'était seulement de 33 p. 100 ef l'ADN cérébral de 24 p. 100 (Potier de Courcy, 1966). Plus spécifiquement, Middaugh ef al. (1976) trouvent une baisse moins importante des folates dans le cerveau de Souris soumise à la carence folique avant la naissance que dans le foie.

4e Réunion du groupe Développement I.N.R.A.

Montpellier, 17-18 mai 1978

Remerciements. - Nous tenons à remercier bien vivement la Cyanamid International (NJ) qui nous a fourni l'acide $X$-méthyl-folique et les établissements Spécia qui nous ont fourni le méthotrexate.

\section{Références}

BARANOV V. S., 1966. The specificity of the teratogenic effect of aminopterin as compared to other teratogenic agents. Bull. exp. Biol. Med. (Moscwa), I, 77-82.

GAULL G. E., VON BERG W., RAIHA N. C. R., STURMAN J. A., 1973. Development of methyltransferase activities of human fetal tissues. Pediat. Res., 37, 527-533.

HERBERT V., 1966. Aseptic addition method for Lactobacillus Casei of folate activity in human serum. J. clin. Pathol., 19, 12-16.

JOHNSON E. M., NELSON M. M., MONIE I. W., 1963. Effects o :transitory pleroyl-glutamic acid (PGA) deficiency on embryonic and placental development in the rat. Anat. Rec., 146, 215-225.

JORDAN R. L., WILSON J. G., SCHUMACHER H. J., 1977. Embryotoxicity of the folate antagonist methotrexate in rats and rabbits. Teratology, 15, 73-79.

LANDON M. J., EYRE D. H., HYTTEN F. E., 1975. Transfer of folate to the foefus. Br. J. Obst. Gynecol., 82, 12-19.

MIDDAUGH L. D., GROVER T. A., BLACKWELL L. A., ZEMP J. W., 1976. Neurochemical and behavorial effects of diet related to perinatal folic acid restriction. Pharmacol. biochem. Behavior, 5 , 129-134.

NAVARRO J., 1973. Valeurs respectives des activités folique, érythrocytaire e folinique sérique comme test de stockage et de métabolisme des folates (chez l'enfant normal ef le prématuré). Arch. franc. Pédiat., 30, 673-683.

NELSON M. M., WRIGHT H. V., ASLING C. W., EVANS H. M., 1955. Multiple congenital abnormalities resulting from transitory deficiency of pteroylglutamic acid during gestation in the rat. J. Nutr., 56, 349-369.

POTIER DE COURCY G., 1966. Conséquences de la carence folique sur les caractères généraux des métabolismes nucléique et protéique chez le fœetus de rat. Arch. Sci. physiol., 20, 189-218.

POTIER DE COURCY G., 1968. Effets biochimiques comparés de trois avitaminoses tératogènes chez la ratte gestante et les fotus. Thèse Doct. Etat, Paris.

POTIER DE COURCY G., BERTAUX O., 1972. Analyse des folates dans les fotus de rat témoins et carencés en acide folique. J. Physiol., 65, $480 \mathrm{~A}$.

POWELL H. R., EKERT H., 1971. Methotrexate-induced congenital malformations. Med. J. Aust., 2, 1076-1077.

THIERSCH J. B., 1952. Therapeutic abortions with a folic acid antagonist 4-aminopteroyl-glutamic acid (4-amino-PGA) administred by the oral route. Amer. J. Obstet. Gynec., 63, 1298-1304

WILSON J. G., 1970. Embryotoxycity of the folic antagonist methotrexate. Anat. Rec., 166, 398. 